\title{
Case - Penile fracture in a patient with Ehlers-Danlos syndrome
}

Mark Biebel, MD ${ }^{1}$; Dayron Rodriguez, MD, MPH${ }^{1}$; Shu Pan, $\mathrm{MD}^{1}$; Alex J. Vanni, MD² ; Ricardo Munarriz, $\mathrm{MD}^{1}$

${ }^{1}$ Boston University School of Medicine, Boston, MA, United States; ${ }^{2}$ Lahey Hospital and Medical Center, Boston, MA, United States

Cite as: Can Urol Assoc J 2019 June 17; Epub ahead of print. http://dx.doi.org/10.5489/cuaj.5982

Published online June 17, 2019

$* * *$

\section{Introduction}

Penile fractures are caused by traumatic rupture of the tunica albuginea surrounding the corpus cavernosum. This injury results in rapid detumescence, penile pain, swelling, and ecchymosis. It is a rare urological emergency and can result in significant morbidity if not repaired expeditiously. Complications include erectile dysfunction, penile deformity/curvature, plaques, painful erections, and infected hematomas. Historically, penile fractures were managed nonsurgically. ${ }^{1,2}$ However, due to complication rates nearing $50 \%$, this is approach has been abandoned in favor of surgical exploration and repair. ${ }^{1-3}$ In the absence of urethral injury, a slight delay in surgical repair (less than one week) has shown no increase in complication rate and allows for resolution of local tissue edema. ${ }^{2,4}$ The incidence of penile fractures is estimated at 1.02 per 100000 males per year, but the true incidence may be underreported. ${ }^{5}$ Patients with connective tissue disorders are more prone to poor surgical outcomes. ${ }^{6}$ Here, we present a case of penile fracture in a patient with Ehlers-Danlos syndrome, which to the best of our knowledge has never been presented in the literature.

\section{Case report}

A 40-year-old man with classical type Ehlers-Danlos syndrome presented to urology clinic 24 hours after he was vigorously masturbating, felt a "pop," and experienced immediate penile detumescence. He had significant ecchymosis and swelling with a palpable hematoma at the ventral, midline base of the penis. He did not have gross hematuria or difficulty voiding. He was taken to the operating room two days later for penile exploration and was found to have bilateral ventral corpora cavernosa injuries. The adjacent urethra was mobilized and inspected circumferentially, revealing no evidence of urethral injury (Figure 1,2). In planning our repair, we considered the AUA guidelines and our typical practice of repairing the tunica with 
absorbable suture. However, based on literature in other surgical fields regarding Ehlers-Danlos syndrome patients and their prolonged wound healing time, the tunical defects were closed using non-absorbable 3-0 Ethibond sutures. ${ }^{6-12}$ Buck's and Dartos fascia were closed in a multi-layer fashion using interrupted 3-0 Monocryl sutures. The skin was closed using 3-0 Nylon sutures in an interrupted horizontal mattress fashion. The non-absorbable skin sutures were removed in clinic on post-operative day 20 and he was instructed to avoid any sexual activity for two additional months. Unfortunately, the patient presented to an out-of-state hospital five months after initial repair with recurrent penile fracture from sexual intercourse. He underwent a repeat penile fracture repair of an injury at the ventral base of the left corpus cavernosum.

Intraoperative cystoscopy confirmed no urethral injury. This surgery was complicated by post-operative urethrocutaneous fistula three weeks later. He returned to the operating room and a dorsal urethral injury was identified adjacent to the site of the most recent tunical defect. The urethra was repaired primarily using 4-0 PDS. The urethral repair failed after removal of the urethral catheter and progressed to a penoscrotal abscess. At this point he was transferred back to our institution and managed with broad spectrum antibiotics and suprapubic catheter urinary diversion. Cystourethroscopy after resolution of the abscess revealed a 1-2 cm dorsal urethral defect. There were visible sutures from the prior repair that appeared to be hindering the healing process (Figure 3). He was taken to the operating room for cystoscopic laser excision of the visible sutures to promote urethral healing. As much suture material was removed as possible without further disruption of the urethral defect, but some deeper suture material remained (Figure 4). Given the high complexity of his injury and underlying connective tissue disorder, the patient was referred to a reconstructive urologist for potential urethrocutaneous fistula repair. At the time of scheduled reconstructive surgery (three months after the suprapubic tube placement), a retrograde urethrogram and cystoscopy revealed spontaneous healing of the urethral fistula. The suprapubic tube was removed and no urethroplasty was necessary.

\section{Discussion}

Ehlers-Danlos syndrome is a heritable group of disorders that affects connective tissues supporting the skin, bones, blood vessels, and many other organs. Common manifestations of the classical type include joint hypermobility, hyperelasticity, and fragile skin. Genetic mutations affecting collagen processing and production are the most common underlying cause. ${ }^{7}$ Poor wound healing and formation of atrophic scars occur frequently in Ehlers-Danlos patients and are two clinical features pertinent to surgical planning. ${ }^{8}$

Penile fracture in a patient with Ehlers-Danlos syndrome has never been reported, perhaps due to the rarity of each pathology. Given this patient's large injury involving bilateral corpora cavernosa in the setting of masturbation, it is reasonable to suspect that his underlying disorder contributed to the severity of his injury and subsequent recurrence. 
Based on our surgical experience and a review of the literature available in other surgical fields, ${ }^{6,9-11}$ we believe surgical outcomes in men with penile fractures and Ehlers-Danlos syndrome (or other connective tissue disorders) can be improved by taking the following into consideration:

1. Use gentle manipulation of tissues. Special care should be taken to handle tissues and particular attention must be given to the skin. Skin retractors should be minimized to avoid traumatizing the skin. Surgical closure may be difficult because sutures may tear out of the wound edges due to the fragility of tissues. ${ }^{6,10-11}$

2. Repair the tunical defect using long-lasting or non-absorbable sutures in an interrupted fashion to avoid dehiscence and to maximize recovery of tissue tensile strength. ${ }^{6,11}$

3. Close the wound in multiple layers with long-lasting, absorbable sutures. ${ }^{6,9-11}$

4. Approximate skin with non-absorbable sutures and keep them in situ for at least 3-4 weeks to avoid skin dehiscence. ${ }^{6,10-11}$

5. Obtain meticulous hemostasis during surgery. Monitor wounds closely for hematoma development post-operatively. Prophylactic anticoagulation (Heparin or Enoxaparin) should be considered with caution, particularly in the Ehlers-Danlos vascular type. ${ }^{6,11}$

6. The typical recommendation for abstinence after penile fracture repair is at least six weeks. ${ }^{12}$ Counsel patients with connective tissue disorders to avoid sexual activity for at least 12 weeks after a penile fracture repair to maximize wound healing.

Despite the use of these techniques, our patient's post-operative course of healing was complex. He had a recurrent penile fracture and associated urethrocutaneous fistula, requiring multiple procedures. This complicated course indicates the need for further research into the relationship between connective tissue disorders and penile fractures, including the optimal surgical techniques and postoperative care instructions. 


\section{References}

1. Amer T, Wilson R, Chlosta P, et al: Penile Fracture: A Meta-Analysis. Urol Int 2016; 96:315-29.

2. Falcone M, Garaffa G, Castiglione F, et al. Current Management of Penile Fracture: An Up-to-Date Systematic Review. Sex Med Rev 2018; 6:253-60.

3. Orvis BR, McAninch JW. Penile rupture. Urol Clin North Am 1989; 16: 369-75.

4. Phillips EA, Esposito AJ, Munarriz R. Acute Penile Trauma and Associated Morbidity: 9-Year Experience at a Tertiary Care Center. Andrology 2015; 3:632-36.

5. Rodriguez D, Apoj M, Munarriz R, et al. Epidemiology of Penile Fractures in United States Emergency Departments: Access to Care Disparities May Lead to Suboptimal Outcomes. J Sex Med 2019;16:248-56.

6. Shirley ED, Demaio M, Bodurtha J. Ehlers-Danlos Syndrome in Orthopaedics: Etiology, Diagnosis, and Treatment Implications. Sports Health 2012; 4:394-403.

7. Genetics Home Reference. Ehlers-Danlos Syndrome. Genetics Home Reference. Accessed May 20, 2018. https://ghr.nlm.nih.gov/condition/ehlers-danlos-syndrome.

8. De Paepe A, Malfait F. The Ehlers-Danlos Syndrome, a Disorder with Many Faces. Clinical Genetics 2012; 82:1-11.

9. Malfait F, Wenstrup RJ, De Paepe A. Clinical and Genetic Aspects of Ehlers-Danlos Syndrome, Classic Type. Genetics in Medicine 2010; 12:597-605.

10. Gupta A, Kumar P. Possible Simple Measures for Complex Wound Healing Problems in Ehlers-Danlos Syndrome. Plast Reconstr Surg Glob Open 2014 Oct; 2(10). https://journals.lww.com/prsgo/Fulltext/2014/10000/Possible_Simple_Measures_for_Co mplex_Wound_Healing.21.aspx Accessed September 3, 2018.

11. Joseph AW, Joseph SS, Francomano CA, et al. Characteristics, Diagnosis, and Management of Ehlers-Danlos Syndromes: A Review. JAMA Facial Plast Surg 2018; 20:70-5.

12. Rees RW, Brown G, Dorkin T, et al. British Association of Urological Surgeons (BAUS) Consensus Document for the Management of Male Genital Emergencies - Penile Fracture. BJU Int; 122: 26-28 
Figures and Tables

Fig. 1. Suture line across bilateral corpora defect.

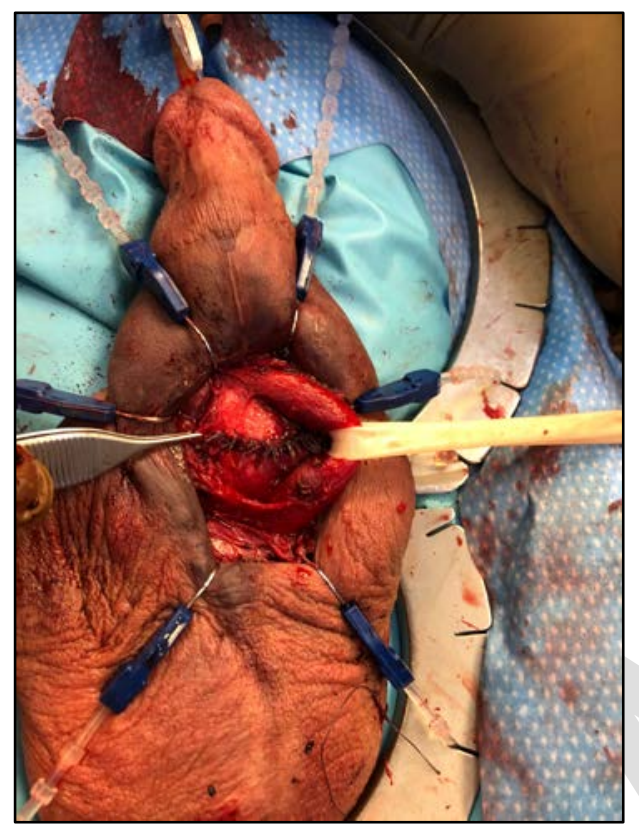

Fig. 2. Urethra and corpus spongiosum mobilized.

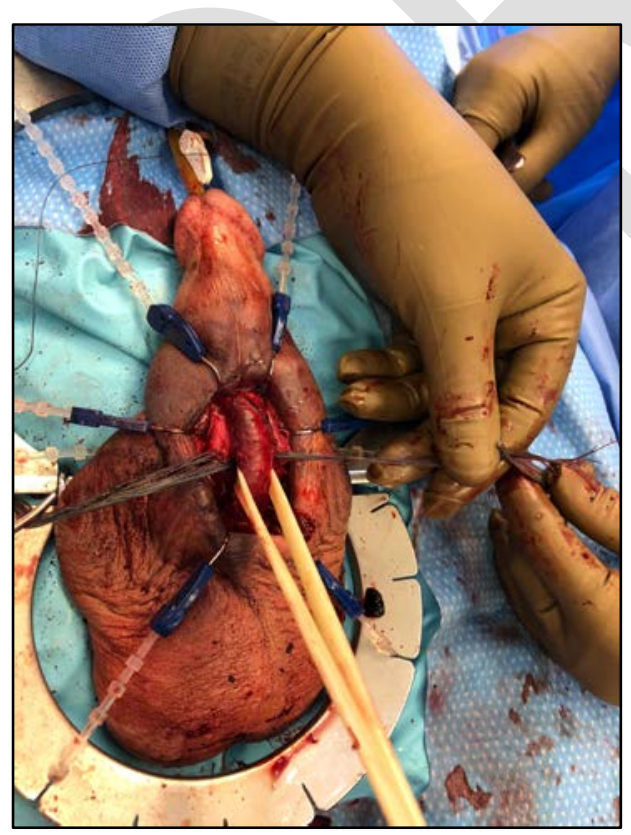


Fig. 3. Cystoscopic image of intraurethral absorbable suture material.

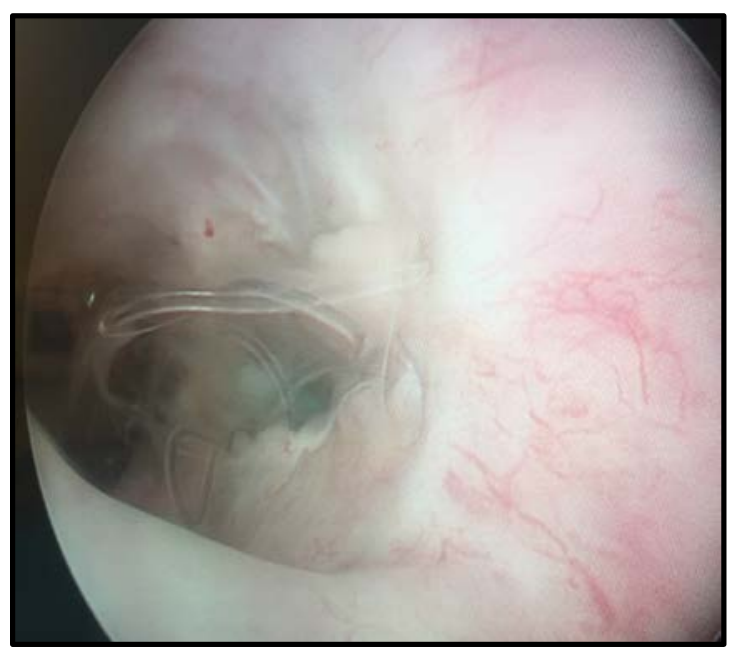

Fig. 4. Cystoscopic image of intraurethral permanent suture material after laser excision.

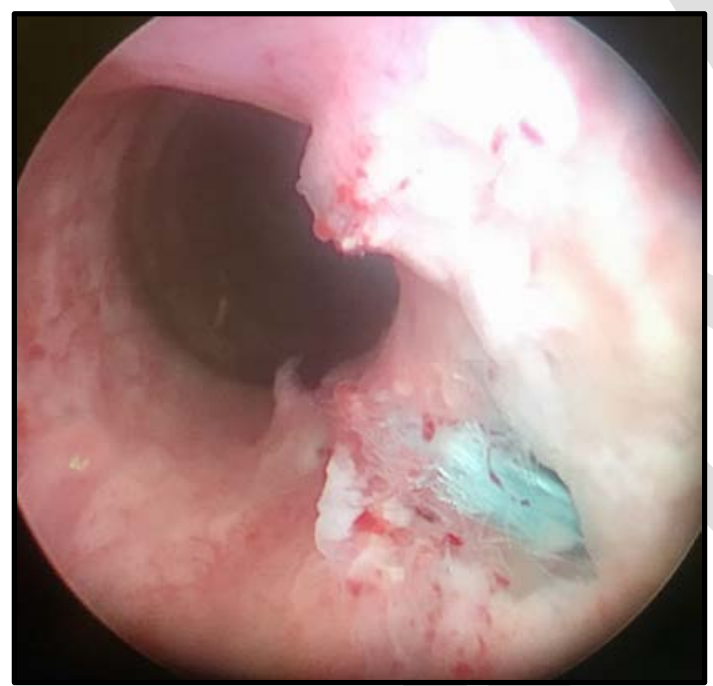

